\title{
A Simple, Practical Method for Measurement of Fat in Milk, Applied to Mid- to Late-Lactating Working Elephants in Myanmar
}

\author{
Yadana Aung Myo Han ${ }^{1,2}$, Ellen S Dierenfeld ${ }^{3 *}$, Khyne U Mar ${ }^{4}$, Mirkka Lahdenperä5 ${ }^{5}$ Virpi Lummaa ${ }^{5}$ and Aung Aung $^{2}$ \\ ${ }^{1}$ Department of Medical Research (Pyin-Oo-Lwin Branch), Myanmar
}

${ }^{2}$ Department of Physiology \&Biochemistry, University of Veterinary Science, Myanmar

${ }^{3}$ LLC, St. Louis, MO USA

${ }^{4}$ New York, NY USA

${ }^{5}$ Department of Biology, University of Turku, Finland

*Corresponding author: Ellen S Dierenfeld, LLC, St. Louis, USA

Submission: July 09, 2018; Published: August 16, 2018

\section{Introduction}

Determining nutrient composition of milk is not only of interest to dairy industry, but may play a key role improving maternal well-being and offspring health and survival in a range of species, including endangered populations of mammals managed in captivity. However, in field conditions, lack of transportation, reagents, equipment, and electricity may limit the applicability of methodologies developed for dairy livestock species to wildlife. One example of a population benefitting from a practical method to assess milk quality in the field is that of Asian elephants employed in timber industry in Myanmar. A third $(\sim 15,000)$ of the remaining, endangered population of Asian elephants lives in captivity in range countries [1]. The largest ( $\sim 5000)$ such captive population is employed in the timber logging industry in Myanmar of which over half belong to the government-owned Myanma Timber Enterprise. However, high calf mortality poses a risk to the population viability: of the identified causes of calf mortality, malnutrition caused by agalactia (lack of or deficient milk production of mothers), was the death reason for $26.3 \%$ of calves dying before age 5 years [2]. Seasonal variation in climate, body condition and mortality rate may exacerbate such effects [3].

Elephant calves are solely dependent on receiving nourishment from their mother's milk for the first months, but poor milk composition can increase calf mortality risk even up to two years or more [2], and lactation usually lasts at least 3-4 years or until the mother gives birth again. Milk quality, measured e.g. by fat content, can vary between mothers of differing condition (e.g. stress levels, age, body condition) and with infant characteristics (e.g. sex, age, birth-order) in elephants [2]. It is therefore important to develop better field methods to assess milk composition in order to identify rapidly which calves are at higher risk of malnutrition and require intervention.

During late pregnancy and throughout lactation, logging elephants owned by the Myanma Timber Enterprise are managed separately from the working herd in maternity camps, enabling training, observation, and research opportunities that target improved management, health, and calf survival. Using longitudinally collected milk samples from these timber elephant females across several seasons of the year, here we describe a modification of a rapid, economic method that has previously been used for estimating fat and energy content in livestock [4] and human [5] milks, for ultimate field application. We also investigate between-female differences in milk fat content, and whether milk samples collected during different seasons from the same females differed in their fat content.

\section{Methods}

To first test the accuracy of the methodology on samples with known fat content, fresh milk and whipping cream samples from local dairy cows in Myanmar were thoroughly mixed and $\sim 75 \mu \mathrm{l}$ aliquots $(n=8)$ were drawn by capillary action into standard glass capillary tubes $(75 \mathrm{X} 15 \mathrm{~mm}$ outside diameter) that were sealed by clay. Tubes were centrifuged at 12,000rpm (approximately 14,500g) for 15 min using a hematocrit centrifuge (Clay Adams Autocrat model CT-2905, New York, NY USA), then immediately the fat layer(s) at the top were measured to the nearest $0.01 \mathrm{~mm}$ using digital calipers (Power fix model Z22855, Paget Trading Ltd., London, UK), and expressed as a percentage of the total milk 
column, following the details in Lucas et al. [5]. Measurement of the solid fat layer meniscus was measured at the top, rather than bottom due to opacity. If clear, liquid fat was present at the top of the cream layer, that fraction was also included with the fat measurement. Measured values compared favourably with known crude fat content of dairy products in Myanmar (3.39 to 4.25\% for milk; $56.67 \%$ for whipping cream), confirming the accuracy of the method.

Second, in total 36 longitudinally collected milk samples (5 to $20 \mathrm{ml}$ ) from six mature Asian elephant (Elephas maximus) cows in mid- (16 mo) to late lactation (38 mo) and living in Katha, Northern Myanmar were obtained on six separate dates over a nine month period between the hot wet season (Jul through Sep 2016; n=18) and cold dry season (Oct 2016 through Mar 2017; n=18). These females were 24, 26, 31, 35, 40 and 41 years old and ranged in parity between two and four, with two of the mothers lactating female calves and four lactating male calves. Sampled were frozen upon collection until analysis in the nutrition laboratory at the Department of Physiology and Biochemistry, University of Veterinary Science, Yezin. Elephant milk aliquots were blended by inversion, and measured in duplicate to obtain "creamatocrit" and liquid fat percentages as per the protocol described above. One sample was determined to be an outlier, and not used in further analyses. Descriptive statistics, paired t-comparisons of means (wet vs. dry season), and one-way analysis of variance were conducted using SPSS (Version 20.0, Chicago, IL).

\section{Result}

Centrifuged elephant milk samples separated into 3 distinct layers: the bottom aqueous fraction, presumably containing proteins, carbohydrates, and soluble minerals, a dense white fat layer, and a clear liquid lipid layer (Figure 1). Percentage lipid fractions in Myanmar elephant milk samples, using the simplified "creamatocrit" method, are found in Table 1 (15.35 mean \pm 4.44 SD\%). The liquid fat layer accounted for $78.88 \pm 12.52 \%$ (range 30.99 to $97.14 \%$ ) of the total fat, as opposed to the denser cream layer (21.11 $\pm 12.52 \%$; range 2.86 to $69.01 \%$ ). The large liquid fat layer in elephant milk differs substantially from results using this method with human or bovine milk samples, where the liquid layer was non-existent or comprised a minor proportion [5]; this study). Total fat content ranged from 7.20 to $26.47 \%$ in milk samples over the 9 months of this sampling period. Significant effects of individual animal, associated with stage of lactation, were found (Table 2); cow age and/or parity effects were not statistically significant. Seasonality was also not significant in this dataset; the mean wet season fat values $(15.57 \pm 1.47 \%$; $=17$ samples from 6 cows) were identical to values measured through the dry season $(13.52 \pm 3.29 \%, n=18$ samples from 6 cows). However, if lag effects due to diet difference are considered, fat content of milk samples from the beginning of the dry season (end of wet season, October; $17.37 \pm 2.05 \%$ ) differed significantly from samples collected at the end of the dry season $12.77 \pm 3.05 \%$; $<<0.01$ (Figure 2).

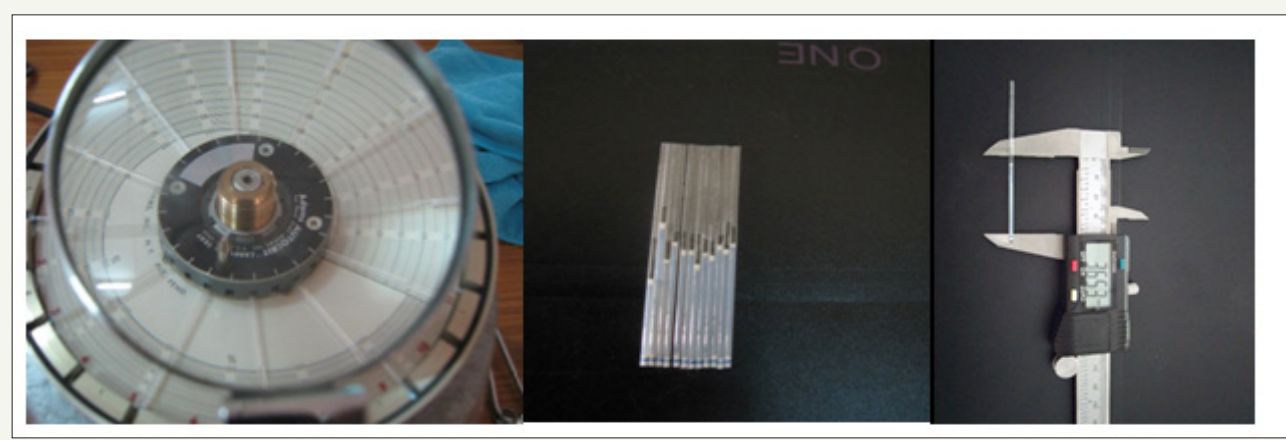

Figure 1: Measuring milk fat in elephant milk samples (as a percentage of total sample) using a simplified centrifugation technique and calipers, suitable for field sampling.

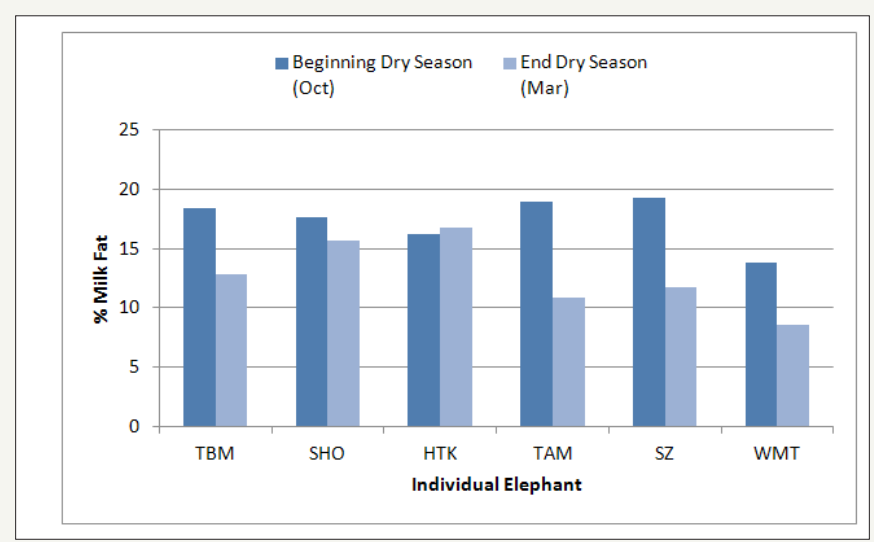

Figure 2: Total milk fat percentages from semi-free ranging Asian elephants $(n=6)$ in Myanmar sampled at beginning (Oct 2016) (Sep/Oct 2016) and end of dry season(Mar 2017), between 19 and 38 months of lactation. 
Table 1: Percentage of total milk fat in Asian elephant milk samples, measured using the "creamatocrit" methodology; both solid and liquid lipid fractions were apparent in all samples.

\begin{tabular}{|c|c|c|c|c|c|}
\hline Description & N & Minimum & Maximum & Mean & 15.35 \\
\hline Total Fat \% & 35 & 7.2 & 26.47 & 21.11 & 4.44 \\
\hline Cream \% Total Fat & 35 & 2.86 & 69.01 & 78.88 & 12.52 \\
\hline Liquid \% Total Fat & 35 & 30.99 & 97.14 & 12.52 \\
\hline
\end{tabular}

Table 2: Variation of milk fat composition from individual Asian elephants in Myanmar, sampled between 16 and 38 months of lactation.

\begin{tabular}{|c|c|c|c|c|c|c|c|}
\hline Animal & $\mathbf{1}$ & $\mathbf{2}$ & $\mathbf{3}$ & $\mathbf{4}$ & $\mathbf{5}$ & $\mathbf{6}$ & $\mathbf{P}$ value \\
\hline Mo of Lactation & $\mathbf{1 6 - 2 4}$ & $\mathbf{1 8 - 2 5}$ & $\mathbf{1 7 - 2 5}$ & $\mathbf{3 0 - 3 8}$ & $\mathbf{2 5 - 3 3}$ & $\mathbf{2 2 - 3 0}$ & $15.36 \pm 4.96$ \\
\hline Milk fat, \% & $14.56 \pm 4.78$ & $16.14 \pm 2.55$ & $15.63 \pm 7.08$ & $12.73 \pm 3.44$ & $16.23 \pm 2.90$ & 0.001 \\
\hline \% Cream fat & $15.93^{\mathrm{d}}$ & $33.97^{\mathrm{a}}$ & $29.52^{\mathrm{b}}$ & $17.93^{\mathrm{d}}$ & $22.54^{\mathrm{c}}$ & $14.9^{\mathrm{d}}$ & 0.001 \\
\hline \% Liquid fat & $85.07^{\mathrm{a}}$ & $71.36^{\mathrm{b}}$ & $72.48^{\mathrm{b}}$ & $79.40^{\mathrm{ab}}$ & $85.90^{\mathrm{a}}$ & $87.93^{\mathrm{a}}$ & 0.001 \\
\hline
\end{tabular}

\section{Discussion}

There is a need for developing easy field methods for milk quality analysis to identify mothers and offspring which are in need of supplementation or human intervention in captive and semi-captive animal populations. Due to limitations on in-country laboratory capabilities to assess milk fat content in Asian elephant mothers in an endangered population in Myanmar, we evaluated total fat content using a low-technology practical method that could also be transferred to the field for immediate monitoring of milk quality, and potential nutritional interventions if necessary.

In general, the protein and fat contents of elephants' milk increase over lactation time [6] demonstrated distinct changes in milk fat content throughout lactation in the same individuals $(n=3$ cows), following the same pattern as reported for African elephants by Mc Cullagh \& Widdowson [7]. For captive-fed Asian elephants, milk samples taken at $\leq 9$ mo of lactation contained $<\sim 13 \%$ fat, whereas samples taken from 18 to $30 \mathrm{mo}$ of lactation contained $15-20 \%$ fat. Therefore, while the documented overall mean total fat values $(15.35 \pm 4.44 \%)$ in our study average considerably higher than those reported by [8] of $7.6 \pm 2.6 \%$ (1994; $n=1$ cow) during the first 9 mo of lactation for Asian elephants, Simon \& Peters $[9,10]$ reported levels varying from 0.95 to $19.0 \%$ during the first $18 \mathrm{mo}$ of lactation. As the Myanmar samples were all from mid- to latelactation cows, our data fit well with these described patterns and confirm high fat content in late lactation milk from Asian elephants.

Although stage of lactation impacted milk fat content in logging camp elephants, there was no significant effect of seasonality in this sample if one defines seasons strictly by calendar months. Nonetheless, milk fat decreased significantly from the beginning to the end of the dry season, with individual cows (4 of 6) displaying end of season fat values $\sim 60$ to $70 \%$ those seen 5 months earlier, likely due to nutritive changes in available vegetation, associated with declining body weight [3] and/or hydration status of the animals. In the case of the latter, however, one might expect milk fat to increase or not change, since higher milk fat has been considered a physiologic mechanism for water conservation in elephants [6].
Consistent seasonal (environmental) effects and potential impacts on nursing calves need to be examined through continued longterm monitoring programs of the elephant herds.

Mc Cullagh \& Widdowson [7] previously reported that elephant (African; Loxodonta africana) milk lipid globules are half the size of bovine milk fats, with a unique fatty acid signature; fatty acid details of Asian elephant milk have not been published [11] confirmed a high content $(\sim 70 \%)$ of short-chain saturated fatty acids (FAs), particularly caprice and lauric acids, low levels of polyunsaturated FAs, and omega-3:omega-6 FA ratios of approximately 1:1 in midlactation African elephant milk, with increasing degree of short chain FA as lactation progresses. Regulatory mechanisms for this process are not yet defined, nor have they been examined in Asian elephant milks. While smaller and shorter chain lipid molecules may be more liquid at room temperatures, the observation of widely ranging proportions of both liquid and solid fat fractions in the centrifuged elephant milk samples need to be investigated further [12]. It is tempting to speculate that the highest proportion of liquid lipid fractions, found in samples from the 2 cows with the oldest calves, may represent this pattern of increased short chain FA in later lactation milks, but no clear pattern of variation (by individual, stage of lactation, month, or season) was discernable in this limited data set.

Most importantly, however, this study documents that reliable fat values for milk samples can be quantified rapidly and economically using this methodology. In the field, it can be very difficult to collect substantial quantities of milk for detailed analysis. By using the small volume necessary for this technique, we can quickly assess dam milk quality through fat content and calculate energy values, while minimizing removal of essential nutrients for the growing calf.

\section{Acknowledgement}

We thank Doug Perni koff, DVM, for his assistance in obtaining the hematocrit centrifuge. Research grants for travel (ESD) and milk analyses were supplied by the Kone Foundation and the World Academy of Sciences. 


\section{References}

1. Sukumar R (2006) A brief review of the status, distribution and biology of wild Asian elephants Elephas maximus. Int Zoo Yb 40:1-8.

2. Mar KU, Lahdenperä M, Lummaa V (2012) Causes and correlates of calf mortality in captive asian elephants (Elephas maximus). PLoS ONE 7(3): e32335.

3. Mumby HS, Mar KU, Thitaram C, Courtiol A, Towiboon P, et al. (2015) Stress and body condition are associated with demography of Asian elephants. Conserv Physiol 3(1):

4. Fleet IR, Linzell JL (1964) A rapid method of estimating fat in very small quantities of milk. Journal of Physiology 175: 15-17.

5. Lucas A, Gibbs JAH, Lyster R, Baum JD (1978) Creamatocrit: simple clinical technique for estimating fat concentration and energy value of human milk. Br Med J1(6119): 1018-1020.

6. Abbondanza FN, Power ML, Dickson MA, Brown J, Oftedal OT (2013) Variation in the composition of milk of Asian elephants (Elephas maximus) throughout lactation. Zoo Biol 32(3): 291-298.
7. McCullagh KG ,Widdowson EM (1970) The milk of the African elephant. Br J Nutr 24: 109.

8. Mainka SA, Cooper RM, Black, SR, Dierenfeld ES (1994) Asian elephant (Elephas maximus) milk composition during the first 280 days of lactation. Zoo Biology 13: 389-393.

9. Simon KJ (1959) Preliminary studies on composition of milk of Indian elephants. Ind Vet J 36: 500-503.

10. Peters JM, Maier R, Hawthorne BE, Storvick CA (1972) Composition and nutrient content of elephant (Elephasmaximus) milk. Journal of Mammalogy 53(4): 717-724.

11. Osthoff G, De Wit M, Hugo A, Kamara BI (2007) Milk composition of three free-ranging African elephant (Loxodonta africana africana) cows during mid lactation. Comp Biochem Physiol B Biochem Mol Bio 148(1): $1-5$.

12. AOAC (1990) Official methods of analysis, (15 th edn),Washington DC, USA.
Creative Commons Attribution 4.0 International License

For possible submissions Click Here

\section{Submit Article}

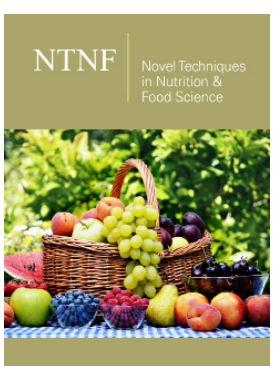

Novel Techniques in Nutrition and Food Science

\section{Benefits of Publishing with us}

- High-level peer review and editorial services

- Freely accessible online immediately upon publication

- Authors retain the copyright to their work

- Licensing it under a Creative Commons license

- Visibility through different online platforms 